Paediatr Paedolog 2016 • [Suppl 1]: 51:S1-S4 DOI 10.1007/s00608-016-0382-8

Online publiziert: 7. Juni 2016

๑) Springer-Verlag Wien 2016

CrossMark

\author{
Johann Deutsch ${ }^{1}$ Sonia Gobara ${ }^{2}$ Franz Waldhauser \\ 'Politische Kindermedizin, Graz, Österreich \\ ${ }^{2}$ Politische Kindermedizin, St. Pölten, Österreich \\ ${ }^{3}$ Politische Kindermedizin, Wien, Österreich
}

\title{
Transition - persönliche Initiativen oder systematisiertes Vorgehen?
}

Unter Transition versteht man die strukturierte, absichtliche und geplante Übergabe eines jugendlichen Patienten bzw. einer Patientin, inklusive einer entsprechenden Schulung, an die Erwachsenenmediziner. Dies unterscheidet sich deutlich von einem Patiententransfer, d.h. von einer lediglich administrativen Übergabe oder Überweisung. Bei Diabetologen oder Hämostaseologen ist dies teilweise gut etabliert, wie sieht es aber mit anderen Erkrankungen bzw. Fachrichtungen aus? Immerhin sind - international gesehen - bis zu 40-60\% der Jugendlichen mit chronischen Erkrankungen in dieser Lebensphase nicht ausreichend betreut [1]. Ein Erkrankungsbeginn im Kindesalter bedingt, dass primär die Eltern oder andere Betreuungspersonen (wie Kindergärtner und Lehrer) bezüglich der Therapie geschult werden. Erst mit zunehmendem Alter können die Jugendlichen in die Betreuung der eigenen Erkrankung allmählich einbezogen werden. Dazu kommen noch die Schwierigkeiten des Jugendlichen bei der Abnabelung vom Elternhaus und die noch nicht abgeschlossene Berufsausbildung, die meist gleichzeitig erfolgen müssen. Als Erwachsener übernimmt der betroffene Patient i. d. R. die Therapie seiner Erkrankung selbst und hat auch die Verantwortung für die Wiedererlangung seiner Gesundheit, ausgenommen im Fall psychischer Erkrankungen, wo Angehörige zumindest zeitweise die Betreuung übernehmen müssen.

Das vorliegende Heft über „Transition“ bringt den Großteil von Vorträgen der ersten Tagung, die sich in
Österreich ausschließlich mit diesem Thema beschäftigt hat [2], und wird ergänzt durch einen Bericht über das von der Gesellschaft für Rheumatologie empfohlene und idealerweise angestrebte Vorgehen. Leider konnten C. Male, T. Michael und M. Pearce ihre Beiträge zur Tagung nicht für diese Publikation zur Verfügung stellen.

\section{) Transition ist schwierig, wenn das Know-how im Erwachsenenbereich fehlt}

Die Ergebnisse der Tagung und die dargestellten Empfehlungen sprechen eindeutig für ein systematisiertes Vorgehen, um zu verhindern, dass unsere jugendlichen Patienten am Beginn des Erwachsenenalters in eine für sie kritische Situation geraten. Die Beiträge dieses Hefts - wie auch die Tagung - zeigen klar auf, dass dieser Prozess i. d. R. dort unproblematisch abläuft, wo genügend Erfahrung, sowohl bei Pädiatern wie auch bei Erwachsenenmedizinern der unterschiedlichen Fachrichtungen, vorhanden sind, z. B. bei den häufigeren Krankheiten im Bereich der Endokrinologie oder Gastroenterologie. Aber auch dort ist eine enge $\mathrm{Zu}$ sammenarbeit zwischen den betroffenen Kollegen und eine etwa 2 Jahre dauernde Schulung der jungen Erwachsenen notwendig, unter den derzeitigen Voraussetzungen sogar ohne zusätzliche finanzielle Ressourcen (wie z. B. für gut vorbereitete gemeinsame Übergabeordinationen). Wesentlich schwieriger ist die Situation in jenen Fällen, wo das Know-how im Er- wachsenenbereich noch nicht vorhanden ist, entweder weil die Erkrankung eine der etwa 8000 seltenen Erkrankungen ist, oder weil bis heute noch wenige Patienten das Erwachsenenalter erreichen. Gerade in dieser Situation sind alle Beteiligten sehr gefordert, sowohl individuell als auch im Bereich der entsprechenden Fachgesellschaften, Ärztekammern und Versicherungen.

Die in diesem Supplementband publizierten Beiträge zeigen einerseits klar auf, wie Transition am besten organisiert wird und zum Wohl unserer Patienten gut funktionieren kann; andererseits werden auch Defizite klar, die derzeit in Österreich noch vorhanden sind. Zum Beispiel haben in Deutschland die Fachgesellschaften für Kinder- und Jugendmedizin und die Gesellschaften für Innere Medizin und Neurologie diesbezüglich ein gemeinsames Vorgehen vereinbart (B. Rodeck), das von fachlicher Seite auch eine gemeinsame Organisation dieses medizinischen Teilbereichs vorsieht; mit den Krankenversicherungen wurde eine Finanzierung der Mehrkosten zumindest als Pilotprojekt vereinbart (S. Müther und J. Findorff). Zusätzlich wurden an verschiedenen Kliniken Deutschlands (E. Koch, S. Müther, B. Rodeck, U. Thyen) und der Schweiz (M. Schwerzmann) entsprechende Projekte der Zusammenarbeit und Schulung (U. Thyen) von Patienten und Ärzten begonnen.

In Österreich existieren verschiedene persönliche Initiativen, z. B. für chronische entzündliche Darmerkrankungen [3] und Hepatologie in Graz, oder für Er- 
krankungen, die auch bei Erwachsenen häufig sind und eine entsprechend intensive Betreuung benötigen, z. B. für Gerinnungsstörungen in Wien. Vereinbarungen zwischen den betroffenen Fachgesellschaften, zwischen Ärztekammern und Versicherungen oder auf der grundlegenden Ebene der Gesundheitsplanung oder Dokumentation fehlen derzeit in Österreich und sollten durch die entsprechenden Organisationen, Gesellschaften und Versicherungen rasch und zielstrebig angegangen werden. Dabei sollte auf die Einbeziehung von Selbsthilfegruppen der Patienten (Beitrag von C. Schneider) und eine Fortführung der Interdisziplinarität besonders Wert gelegt werden.

Die Herausgeber

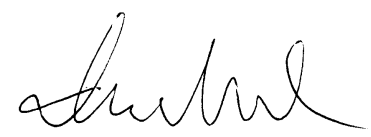

J. Deutsch

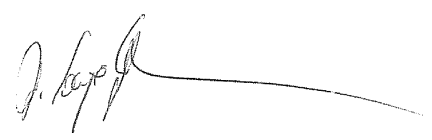

S. Gobara

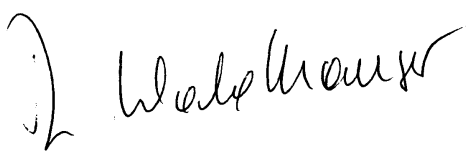

F. Waldhauser

Graz, im Juni 2016

\section{Korrespondenzadresse}

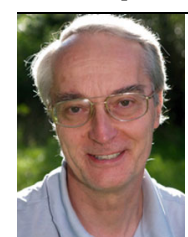

Univ.-Prof. Dr. J. Deutsch

Politische Kindermedizin

Schaftalstrasse 41, $8010 \mathrm{Graz}$,

Österreich

jeejrm@yahoo.de

(c) JMD

Interessenkonflikt. J. Deutsch, S. Gobara undF. Waldhauser geben an, dass kein Interessenkonflikt besteht.

\section{Literatur}

1. McManus MA, Pollack L, Cooley C, McAllister JW, Lotstein D, Strickland B, Mann MY (2013) Current status of transition preparation among youth with special needs in the United States. Pediatrics 131:1090-1097. doi:10.1542/peds.2012-3050
2. Lost in Transition - wenn aus Kindern Erwachsene werden. 9. Jahrestagung der Politischen Kindermedizin, Salzburg 16.-17.10.2015. http://www. polkm.org/meetings/ann_meeting_15/salzb_15. pdf

3. Hauer A (2015) Chronisch Entzündliche Darmerkrankungen und Transition. MSD, Wien 
Hier steht eine Anzeige.

\section{曾 Springer}


Hier steht eine Anzeige.

\section{曾 Springer}

